\title{
Interval versus continuous training in individuals with chronic obstructive pulmonary disease- a systematic review
}

\author{
Marla K Beauchamp, ${ }^{1}$ Mika Nonoyama, ${ }^{2}$ Roger S Goldstein, ${ }^{2}$ Kylie Hill, ${ }^{2}$ \\ Thomas E Dolmage, ${ }^{3}$ Sunita Mathur, ${ }^{4}$ Dina Brooks ${ }^{4}$
}

${ }^{1}$ Graduate Department of Rehabilitation Science, University of Toronto, Toronto, Ontario, Canada ${ }^{2}$ Respiratory Medicine, West Park Healthcare Centre, Toronto, Ontario, Canada ${ }^{3}$ Respiratory Diagnostic and Evaluation Services, West Park Healthcare Centre, Toronto, Ontario, Canada ${ }^{4}$ Department of Physical Therapy, University of Toronto, Toronto, Ontario, Canada

Correspondence to Professor Dina Brooks, Department of Physical Therapy, University of Toronto, 500 University Avenue, Toronto, Ontario, Canada; dina.brooks@utoronto.ca

Received 6 July 2009 Accepted 24 October 2009 Published Online First 8 December 2009
Background In patients with chronic obstructive pulmonary disease (COPD), interval exercise has gained recent attention as a possible means of achieving greater physiological training effects compared with continuous exercise. The primary aim of this systematic review was to compare the effects of interval versus continuous training on peak oxygen uptake, peak power, 6 minute walk test (6MWT) distance and health-related quality of life in individuals with COPD.

Methods Randomised controlled trials comparing the effects of interval versus continuous training in patients with COPD were identified after searches of six databases and reference lists of appropriate studies in May 2009. Two reviewers independently assessed study quality. Weighted mean differences (WMD) with 95\% Cls were calculated using a random effects model for measures of exercise capacity and health-related quality of life.

Results: Eight randomised controlled trials, with a total of 388 patients with COPD, met the inclusion criteria. No significant differences were found for peak power (WMD $1 \mathrm{~W}, 95 \% \mathrm{Cl}-1$ to 3 ) or peak oxygen uptake (WMD $-0.04 \mathrm{l} / \mathrm{min}, 95 \% \mathrm{Cl}-0.13$ to 0.05 ) between interval and continuous training. The WMD for the Chronic Respiratory Questionnaire dyspnoea score was -0.2 units ( $95 \% \mathrm{Cl}-0.5$ to 0.0$)$. There was no difference in 6MWT distance between groups (WMD 4 m, 95\% $\mathrm{Cl}-15$ to 23).

Conclusions Interval and continuous training modalities did not differ in their effect on measures of exercise capacity or health-related quality of life. Interval training may be considered as an alternative to continuous training in patients with varying degrees of COPD severity.

\section{INTRODUCTION}

Chronic obstructive pulmonary disease (COPD) is one of the most common causes of death worldwide and is projected to rank third in 2020 in global burden of disease. ${ }^{1}$ The natural course of COPD is that of progressive airflow limitation, repeated exacerbations, respiratory failure and premature death. In addition to the primary pulmonary pathology, individuals with COPD develop secondary systemic manifestations of the disease including respiratory and peripheral muscle dysfunction, weight loss, osteoporosis, anxiety and depression. These extrapulmonary effects have a significant impact on the quality of life, symptoms and mortality in individuals with COPD. ${ }^{2}$
There is strong evidence that pulmonary rehabilitation increases exercise tolerance, reduces symptoms and improves health-related quality of life in patients with COPD. ${ }^{3}$ Although pulmonary rehabilitation includes education, nutritional, social and psychological support, the physical exercise component is considered the cornerstone of rehabilitation. ${ }^{4}$ While higher intensity training results in greater gains in exercise capacity compared with lower intensity exercise, ${ }^{5}$ many patients with COPD are unable to sustain a high intensity load for the targeted training duration due to intolerable symptoms. ${ }^{67}$ Therefore, there is increasing interest in strategies, such as interval training, that may provide a tolerable training load while maintaining an effective stimulus for adaptation. ${ }^{8-16}$

Interval training protocols of repeated bouts of high intensity exercise interspersed with recovery periods have long been demonstrated to be helpful for improving endurance performance and corresponding physiological variables in recreational and elite individuals. ${ }^{17}{ }^{18}$ Interval training has also gained recent attention in individuals with chronic heart failure as it has been suggested to produce more favourable cardiovascular effects compared with traditional continuous exercise in these patients. ${ }^{19}$

In individuals with COPD, it is proposed that interval exercise may increase the tolerance for high intensity work through recovery periods that facilitate a decrease in end expiratory lung volume as well as a reduction in metabolic products from the working muscle that stimulate ventilation. ${ }^{12} 14$ Several small randomised controlled trials (RCTs) have been conducted in an attempt to determine the most effective training modality for patients with COPD; however, results have been inconclusive. ${ }^{8-11} 13151620$ Therefore, the primary aim of this systematic review was to compare the effects of interval versus continuous training on peak oxygen uptake $\left(\mathrm{VO}_{2 \text { peak }}\right)$, peak power $\left(\mathrm{P}_{\text {peak }}\right)$, 6 minute walk test (6MWT) distance and healthrelated quality of life in individuals with COPD.

\section{METHODS}

\section{Search strategy}

Study identification began with electronic searching of computerised databases (MEDLINE, PubMed, EMBASE, CINAHL, PEDro and Cochrane) from inception until May, 2009. The Medical Subject Headings used in the search were: "pulmonary disease", "chronic obstructive", "lung", "exercise" and "rehabilitation". Key terms for the search included "interval/intermittent training", "continuous 
training", "COPD/obstructive lung disease" and "pulmonary/ respiratory rehabilitation". Secondary searches included hand searching reference lists of all identified studies, key author searches and use of the PubMed "related articles" function. Corresponding authors of included RCTs were contacted for missing data where necessary. All authors who were contacted responded.

\section{Inclusion criteria}

One member of the research team (MB) performed the search and two members (MB and $\mathrm{DB}$ ) assessed the studies. Only randomised trials comparing interval with continuous exercise training using standardised protocols (cycle or treadmill at measurable exercise intensity) were considered for inclusion. More than $90 \%$ of study participants must have been diagnosed with COPD defined as: (1) a clinical diagnosis of COPD; and (2) one of the following (a) best recorded forced expiratory volume in $1 \mathrm{~s}\left(\mathrm{FEV}_{1}\right) /$ forced vital capacity (FVC) ratio $<0.70$ or (b) best recorded $\mathrm{FEV}_{1}$ of $<80 \%$ predicted. ${ }^{21}$ We considered the following outcome measures: $\mathrm{P}_{\text {peak }}$ and $\mathrm{VO}_{2 \text { peak }}$ measured during an incremental exercise test on a cycle ergometer or treadmill; endurance time measured from a constant power test; functional exercise capacity measured by 6MWT or 12MWT; health-related quality of life as measured by the Chronic Respiratory Questionnaire (CRQ); and anxiety and depression measured by the Hospital Anxiety and Depression scale (HAD). In addition, we extracted data on physiological parameters documenting training effects (lactate threshold, isotime ventilation, heart rate, breathing frequency and symptoms), skeletal muscle adaptations and tolerance to training (eg, compliance, adverse effects). The search identified studies of all languages.

Specific exclusion criteria comprised: (1) not an intervention study with an appropriate comparison group; and (2) only an abstract available with no further information from authors.

\section{Quality assessment}

Two members of the review team (MB and DB) independently assessed the internal validity of the studies. The quality assessment was rated according to a $0-5$ scale suggested by Jadad ${ }^{22}$ including items related to randomisation, blinding and withdrawals.

Each trial was also given a score on a 10-point scale using the Physiotherapy Evidence Base Database (PEDro) scale. ${ }^{23}$ The PEDro assesses quality criteria related to blinding, randomisation, withdrawals, comparability of baseline characteristics and data reporting.

\section{Statistical analysis}

Trial data were combined using Review Manager 5.0 (Cochrane Collaboration's Information Management System), with all outcome measures treated as continuous variables. The weighted mean difference (WMD) was selected when estimating the total effect of combined data. The random effects model was used for all analyses, and forest plots were used to depict the results visually. The change due to continuous training was subtracted from the change due to interval training for each outcome; therefore, a positive WMD indicated that the effect for interval training was greater than for continuous training. Heterogeneity was tested with the $\mathrm{I}^{2}$ statistic.

Point estimates and confidence limits for the difference between groups were assessed to see if they exceeded the minimal important difference. We chose 0.5 as the minimal important difference for the CRO domains of dyspnoea and total score, ${ }^{24}$ and $54 \mathrm{~m}$ for the $6 \mathrm{MWT}{ }^{25}$

\section{RESULTS}

\section{Search results}

The study selection process is outlined in figure 1 . Of a possible 578 trials, eight were included in this review. ${ }^{8-11} 13151620$ One study was retrieved as an abstract only ${ }^{26}$ but information was provided by the author and the full manuscript was published at the time of this review. ${ }^{10}$ In total, 388 COPD patients were randomised to either interval or continuous training. Table 1 shows the characteristics of those included in the trials. Patients were elderly (mean 67 years) and had moderate to severe COPD (mean $\mathrm{FEV}_{1}$ 33-55\% predicted). Seven of the eight studies included a majority of male patients, ${ }^{9-11} 13151620$ while one study ${ }^{8}$ included mostly (85\%) female patients.

There was consistent agreement between reviewers for the quality assessment. Study quality ratings based on Jadad and PEDro scales are provided in table 2 . The studies achieved a mean Jadad score of $2 / 5$ (range 1-3) and mean PEDro score of $6 / 10$ (range 5-8). Three trials provided details about randomisation,, 811 three reported allocation concealment ${ }^{8} 10^{11}$ and only two trials ${ }^{10}{ }^{11}$ reported blinding of outcome assessors. Only one study included a specific statement regarding adverse events and reported no adverse events with either type of training. ${ }^{13}$

\section{Training protocols}

Continuous training protocols used moderate to high intensity exercise of $50-80 \%$ of the $\mathrm{P}_{\text {peak }}$, obtained on an incremental test. Exercise duration ranged from 20 to $45 \mathrm{~min}$. Interval training protocols used high intensity exercise $\left(\geq 80 \%\right.$ of $\mathrm{P}_{\text {peak }}$, range $80-150 \%$ ) of $20 \mathrm{~s}-3 \mathrm{~min}$ alternating with low to moderate intensity $\left(30-75 \% \quad \mathrm{P}_{\text {peak }}\right)$ recovery periods of $30 \mathrm{~s}-3 \mathrm{~min}$ (table 1). Two studies ${ }^{9}{ }^{10}$ used 1 min intervals of higher intensity exercise $\left(\geq 90 \% \mathrm{P}_{\text {peak }}\right.$ ) alternating with 2 min of low intensity exercise $\left(\leq 75 \% \mathrm{P}_{\text {peak }}\right)$, three trials ${ }^{15} 1620$ used $30 \mathrm{~s}$ intervals $\left(100 \% / 45 \% \mathrm{P}_{\text {peak }}\right)$, one study ${ }^{11}$ alternated high $\left(50 \% \mathrm{P}_{\text {peak }}\right)$ and

Total citations identified from electronic databases (Medline, PubMed, Embase, Pedro, Cochrane, CINAHL) $n=578$

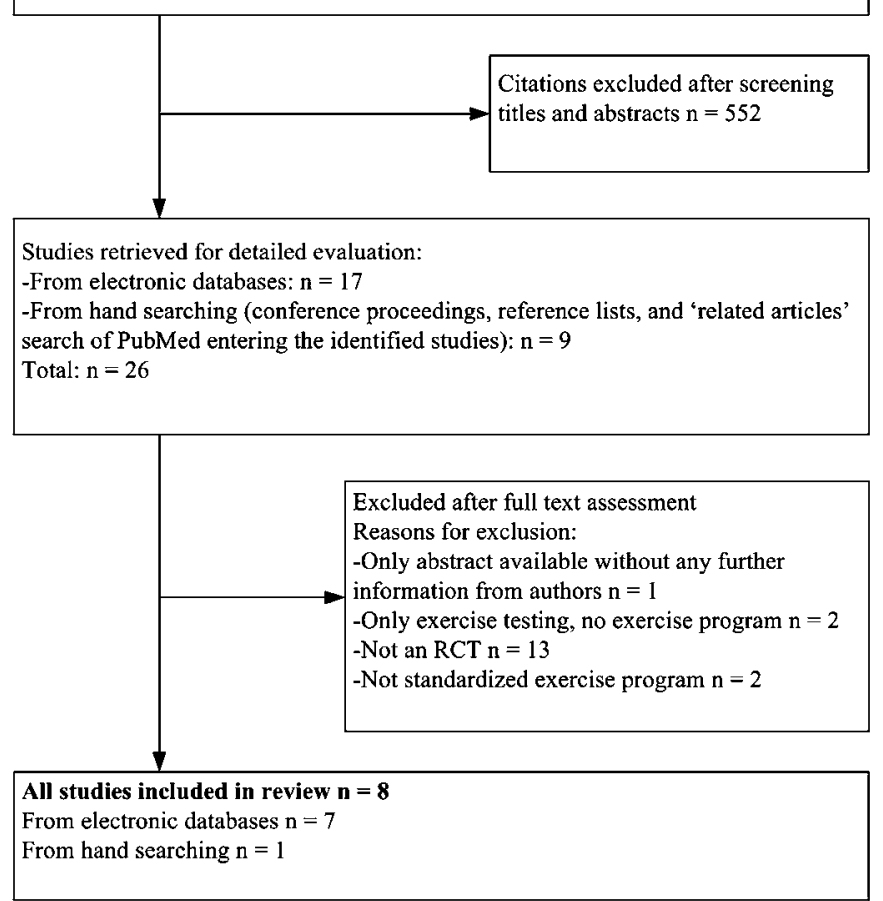

Figure 1 Study flow from identification to final inclusion of studies. 


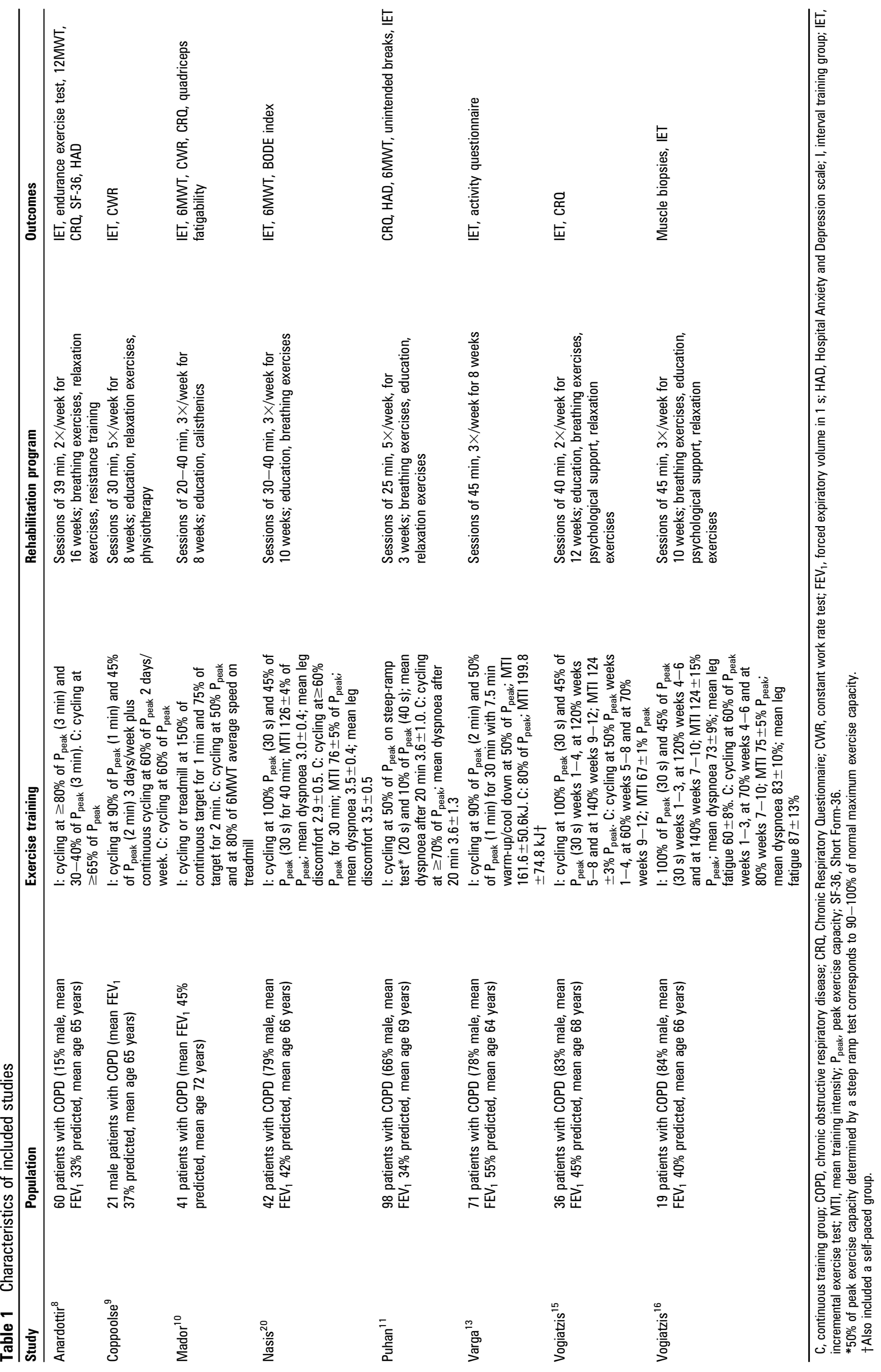


Table 2 Quality assessment results

\begin{tabular}{|c|c|c|c|c|c|c|c|}
\hline Study & Study design & $\begin{array}{l}\text { Description of } \\
\text { randomisation }\end{array}$ & $\begin{array}{l}\text { Allocation } \\
\text { concealment }\end{array}$ & $\begin{array}{l}\text { Description of } \\
\text { withdrawals }\end{array}$ & $\begin{array}{l}\text { Blinding of } \\
\text { outcome } \\
\text { assessors }\end{array}$ & $\begin{array}{l}\text { Jadad } \\
\text { score }\end{array}$ & $\begin{array}{l}\text { PEDro } \\
\text { score }\end{array}$ \\
\hline Anardottir ${ }^{8}$ & Parallel RCT & + & + & \pm & - & $2 / 5$ & $6 / 10$ \\
\hline Mador $^{10}$ & Parallel RCT & + & + & \pm & \pm & $2 / 5$ & $8 / 10$ \\
\hline Nasis $^{20}$ & Parallel RCT & - & - & - & - & $1 / 5$ & $6 / 10$ \\
\hline Puhan $^{11}$ & Randomised non-inferiority & + & + & + & \pm & $3 / 5$ & $8 / 10$ \\
\hline Vogiatzis $^{16}$ & Parallel RCT & - & - & - & - & $1 / 5$ & $6 / 10$ \\
\hline
\end{tabular}

$\mathrm{RCT}$, randomised controlled trial; + , requirement fulfilled; - , requirement not fulfilled or not reported; \pm , requirement partially fulfilled.

low $\left(10 \% \mathrm{P}_{\text {peak }}\right)$ intensities of 20 and $40 \mathrm{~s}$ intervals, and one study ${ }^{13}$ used 2 min intervals of high intensity exercise $\left(90 \% \mathrm{P}_{\text {peak }}\right)$ alternated with 1 min of low intensity exercise (50\% $\left.\mathrm{P}_{\text {peak }}\right)$. Intensity at the start of training was selected as a proportion of $\mathrm{P}_{\text {peak }}$ achieved during a symptom-limited incremental exercise test in seven studies. ${ }^{8-10} 13151620$ In one study, ${ }^{11} \mathrm{P}_{\text {peak }}$ for interval exercise was defined as $50 \%$ of the highest power achieved on a steep ramp test which corresponded to $98 \%$ of $\mathrm{P}_{\text {peak }}$ on an incremental test. ${ }^{27}$

Seven studies $8 \quad 9 \quad 11 \quad 13 \quad 15 \quad 16 \quad 20$ included exercise on cycle ergometers for both training protocols; however, one study ${ }^{10}$ included exercise on either a treadmill or cycle ergometer. In this trial, the speed for treadmill training was determined as a percentage of the average speed elicited during the 6MWT. Total work performed during training was matched at baseline between exercise modes in six trials. ${ }^{9} 1013151620$ In these studies, the progression of training was arbitrarily set in each group. Two studies increased the training load (intensity or duration) on an individual basis and reported that patients in interval training groups showed a trend towards less total work than those trained with continuous exercise. ${ }^{8} 11$

\section{Exercise capacity and response}

$\mathrm{P}_{\text {peak }}$, measured by incremental cycle ergometry, was pooled from all eight included trials (337 patients). The common effect (WMD) in $\mathrm{P}_{\text {peak }}$ was $1 \mathrm{~W}(95 \% \mathrm{CI}-1$ to 3$)$ (figure $\left.2 \mathrm{~A}\right)$. The $\mathrm{WMD}$ for $\mathrm{VO}_{2 \text { peak, }}$, measured in six trials, was $-0.04 \mathrm{l} / \mathrm{min}(95 \%$ $\mathrm{CI}-0.13$ to 0.05 ) (figure $2 \mathrm{~B}$ ). Oxygen uptake at lactate threshold was measured in four trials, and showed a WMD of $0.011 / \mathrm{min}$ (95\% CI -0.05 to 0.07 ) (figure $2 \mathrm{C}$ ).

In all studies, isotime was defined for each individual as the point of termination of the shorter of the baseline and follow-up exercise test; this point most often occurs when the baseline test ends. Isotime minute ventilation $\left(\mathrm{V}_{\mathrm{E}}\right)$ from incremental cycle ergometry was measured in four studies (135 patients) with a WMD of $-0.831 / \mathrm{min}(95 \% \mathrm{CI}-5.01$ to 3.36$)$. The isotime Borg Dyspnoea Scale was pooled from four studies and showed a WMD of -0.3 ( $95 \% \mathrm{CI}-1.0$ to 0.4$)$ between groups. The WMD for isotime breathing frequency from four studies was 0 breaths/ $\min (95 \% \mathrm{CI}-2$ to 2$)$ and isotime heart rate from five studies was 2 beats/min ( $95 \% \mathrm{CI}-3$ to 7$)$.

Two studies (101 patients) used a constant power endurance exercise test and reported isotime responses. A WMD of $-2.26 \mathrm{l} /$ $\min (95 \% \mathrm{CI}-5.59$ to 1.07$)$ was found for $\mathrm{V}_{\mathrm{E}}$. Only one study ${ }^{10}$ reported endurance time (between-group difference $=-3.7 \mathrm{~min}$; $95 \%$ CI -11.8 to 4.4 ).

The effect of the two training modes on walk distance was measured in four trials (228 patients). Three trials used the $6 \mathrm{MWT}$ and one used the 12MWT; as such, data for the 12MWT were divided by two for the purposes of the meta-analysis. The WMD for 6MWT was $4 \mathrm{~m}$ (95\% CI -14 to 23$)$ (figure 3$)$.

\section{Health-related quality of life, anxiety and depression}

The CRO dyspnoea domain was measured in four studies (218 patients). The WMD showed a non-significant trend $(p=0.10)$ of -0.2 units $(95 \%$ CI -0.5 to 0.0$)$ between training modes (figure 4). Total CRO scores were pooled from three studies (162 patients), resulting in a WMD of -0.1 units ( $95 \% \mathrm{CI}-0.3$ to 0.2 ). $\mathrm{HAD}$ scores were pooled from two studies. Common effects for both anxiety and depression subscales were 0.3 (95\% CI -0.8 to $1.3)$ and 0.5 (95\% CI -0.5 to 1.5$)$ units, respectively.

Table 3 summarises the results of the meta-analyses for each outcome. Tests of heterogeneity on all measures of exercise capacity and quality of life were not significant, and $\mathrm{I}^{2}$ ranged from $0 \%$ to $13 \%$, indicating that a low percentage of the variability in effect estimates was due to heterogeneity. Quantitative interpretations were limited because of the few studies available for comparison. The small number of studies also precluded the creation of funnel plots to test for the presence of publication bias. Sensitivity analyses were computed as indicated; however, there were no significant effects on the pooled analysis for any outcome.

\section{Skeletal muscle adaptations}

Two studies considered the effect of interval training on skeletal muscle adaptations compared with continuous exercise. ${ }^{10} 16$ Vogiatzis et al found significantly improved cross-sectional areas of type 1 and type Ila fibres and capillary to fibre ratio in the quadriceps muscle after both interval and continuous training, with no differences between groups. ${ }^{16}$ Similarly, Mador et al noted improvements in quadriceps fatigability following both types of training, with no differences between training modes. ${ }^{10}$

\section{Tolerance to training}

Attendance rates (reported in five studies) ranged from $83 \%$ to $92 \%$, with no differences between training modes. ${ }^{8} 10111315$ Drop-outs did not differ between groups, ranging from no dropouts $^{13}$ to 40 drop-outs in a larger trial $(n=100)$ by Anardottir and colleagues. ${ }^{8}$ The most commonly cited reason for study drop-out was exacerbations (35 participants). ${ }^{811}$ Both trials by Vogiatzis and colleagues ${ }^{1516}$ reported significantly reduced perceptions of dyspnoea and leg discomfort during interval training compared with continuous training; however, two other studies 810 noted no significant differences in symptoms between exercise modes. Puhan and colleagues ${ }^{11}$ reported significantly fewer unintended breaks of $\geq 1 \mathrm{~min}$ in the interval training group (median 2) compared with the continuous training group (median 11). In this study, ${ }^{11}$ adherence to the planned exercise protocol-that is, the proportion of patients who achieved the target exercise 
Figure 2 Comparison of the effect of interval versus continuous training on (A) peak power (Watts), (B) peak oxygen uptake (I/min) and (C) lactate threshold $(\mathrm{l} / \mathrm{min})$. The square on the horizontal line represents the point estimate. The size of the square is determined by how much weight the study contributes to the pooled effect estimate. The diamond represents pooled effect estimates.
A

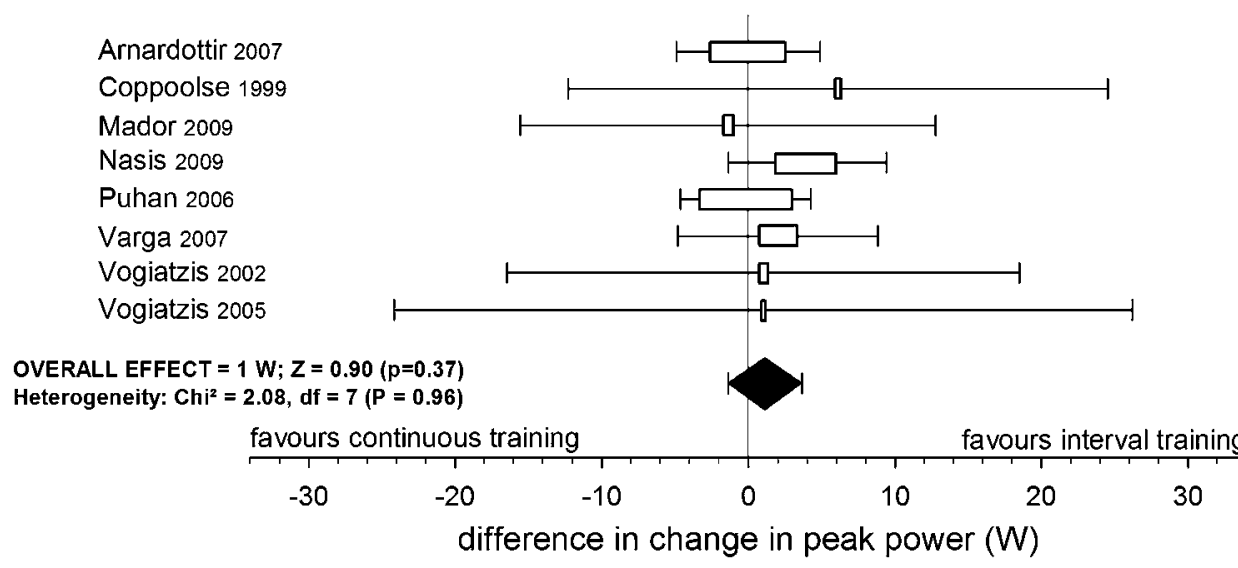

B

Arnardottir 2007
Coppoolse 1999
Mador 2009
Varga 2007
Vogiatzis 2002
Vogiatzis 2005

OVERALL EFFECT $=-0.04 \mathrm{~L} / \mathrm{min} ; \mathrm{Z}=0.94(p=0.35)$ Heterogeneity: $\mathrm{Chi}^{2}=1.36, \mathrm{df}=5(P=0.93)$

favours continuous training

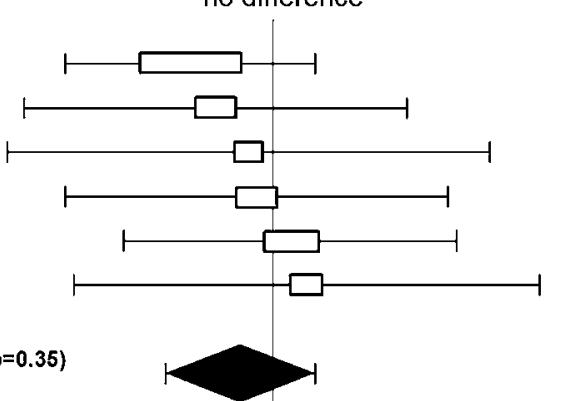

favours interval training
C

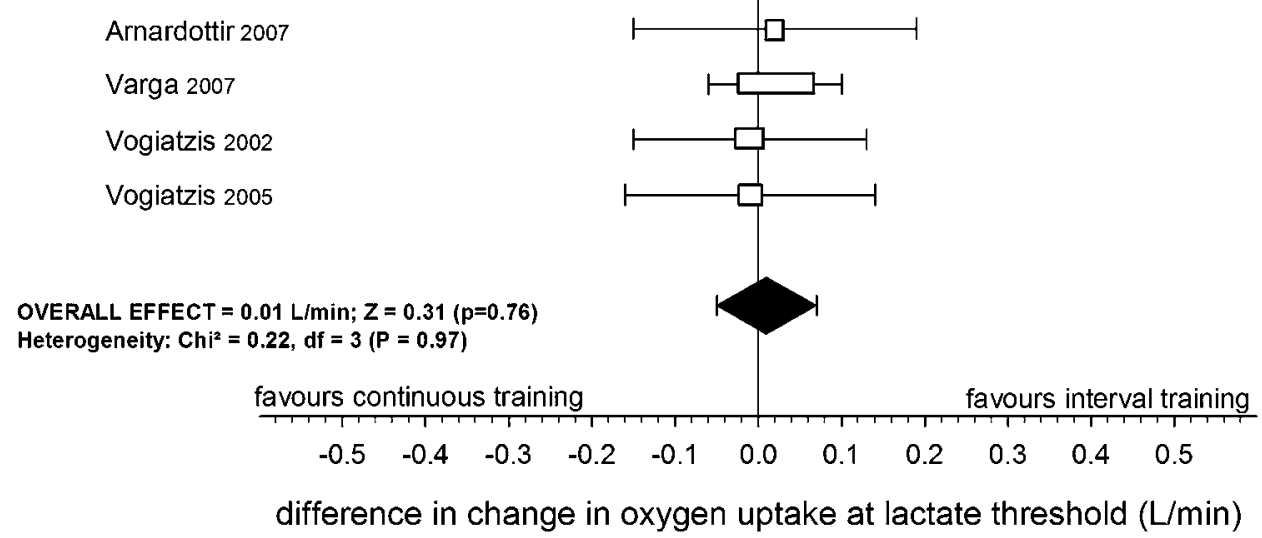

intensity-significantly favoured the interval training group (48\% vs $24 \%)$.

\section{DISCUSSION}

The results of this meta-analysis suggest that there are no differences between the effect of interval and continuous training on measures of exercise capacity or on health-related quality of life in individuals with moderate to severe COPD. While these findings suggest that interval training may be an alternative to continuous training in these patients, limitations in the protocols of the included studies preclude definitive conclusions.

In healthy subjects and in those with chronic heart failure, high intensity interval training has been shown to be as effective as or superior to continuous training with respect to effects on physiological markers of exercise capacity. ${ }^{18} 19$ Although we note that in the eight studies of patients with COPD included in this review, interval training resulted in significant improvements in exercise capacity and health-related quality of life, the metaanalysis does not suggest greater gains in $\mathrm{P}_{\text {peak }}, \mathrm{VO}_{2 \text { peak }}$ or $\mathrm{V}_{\mathrm{E}}$ from interval training compared with continuous endurance training. Two studies also suggest that both training modes elicit similar training responses in the muscles of ambulation. ${ }^{10} 16$

Several factors may have attenuated any differences observed between continuous and interval training approaches in the included studies. In patients with COPD, interval training has gained attention as a potential means of achieving greater 
Figure 3 Comparison of the effect of interval versus continuous training on 6 minute walk distance (metres). The square on the horizontal line represents the point estimate. The size of the square is determined by how much weight the study contributes to the pooled effect estimate. The diamond represents pooled effect estimates.

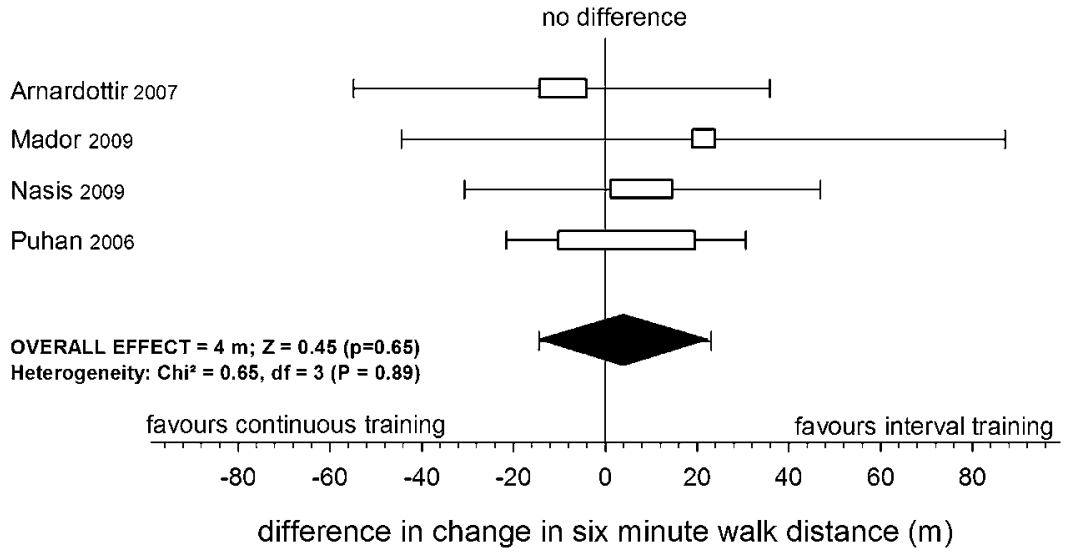

physiological training effects, by increasing the capacity for higher intensity work. ${ }^{121428}$ However, in six 91013151620 of the eight studies included in this review, the total work performed for the interval and continuous exercise trained groups was matched. While this approach allows for comparison of responses after the same total work, the physiological benefits of training are more likely to be apparent when both baseline and progression of exercise intensity are individualised. In the two studies $^{8} 11$ in which the load was individualised to patient tolerance, the interval trained groups performed less total work than the continuous trained groups. Given that total work is an important determinant of training response, future studies are required to determine whether patients with COPD are capable of performing more work at higher intensities with interval training, theoretically leading to greater benefits compared with continuous exercise.

Another confounding factor in the comparison of interval and continuous training was the small absolute difference in power between the high intensity phase of interval training and the continuous load, which ranged from 5 to $15 \mathrm{~W}$. For example, if the average $\mathrm{P}_{\text {peak }}$ from an incremental test is $50 \mathrm{~W}$, a high intensity interval of $80 \% \mathrm{P}_{\text {peak }}(40 \mathrm{~W})$ is only $5 \mathrm{~W}$ greater than a targeted continuous intensity set at $70 \% \mathrm{P}_{\text {peak }}(35 \mathrm{~W})$. Therefore, short duration intervals at relatively moderate intensities probably place the interval group under considerably less physiological stress unless more work is performed. Furthermore, the duration of the exercise programmes in six of the studies ${ }^{9-11} 1316$ ${ }^{20}$ was shorter than the 12 weeks recommended by guidelines for pulmonary rehabilitation. ${ }^{4}$ In unfit individuals, any exercise is likely to be effective $e^{29}$ and it may therefore be difficult to demonstrate large differences between training approaches during relatively short programmes, regardless of which it might favour. Finally, although a high intensity constant power endurance test provides greater sensitivity to discriminate change than an incremental exercise test, ${ }^{30}$ only one study ${ }^{10}$ included this outcome. In this study, the improvement in endurance following continuous training was greater than that following interval training and, although not statistically significant, was more than the clinically meaningful difference. ${ }^{31} 32$

A typical response following an effective exercise regimen is a post-training decrease in ventilation for any given workload. In this review, post-training pooled results for breathing frequency, heart rate, dyspnoea and $V_{E}$ at isotime did not show betweengroup differences for interval versus continuous exercise. However, if the high intensity bouts in interval training provide adequate stimulus for adaptation without causing a ventilatory response, $^{12}{ }^{14}$ this type of training may be better suited for patients with more severe ventilatory limitation. In fact, the mean $\mathrm{FEV}_{1}$ in the two studies ${ }^{816}$ that demonstrated a benefit in favour of interval training was lower ( $\leq 40 \%$ predicted) than in the other trials, providing support for this theory. tolerated and feasible among patients with COPD, with no between-group differences in withdrawals or training attendance. Puhan and colleagues ${ }^{11}$ noted superior adherence to the prescribed training protocol and less frequent rest periods in patients undergoing interval training compared with continuous exercise. However, measuring rest periods in a protocol with built in rest intervals may compromise the validity of this outcome. In contrast to the findings of Mador and Anardottir of no difference in symptoms between training modes, ${ }^{8} 10$ both trials by Vogiatzis ${ }^{15} 16$ reported reduced dyspnoea and leg fatigue during interval training sessions compared with continuous exercise. As the reports of Puhan ${ }^{11}$ and Vogiatzis ${ }^{15} 16$ included the shortest
In all studies, interval and continuous training were both well
Figure 4 Comparison of the effect of interval versus continuous training on the Chronic Respiratory Questionnaire, dyspnoea domain. The square on the horizontal line represents the point estimate. The size of the square is determined by how much weight the study contributes to the pooled effect estimate. The diamond represents pooled effect estimates.

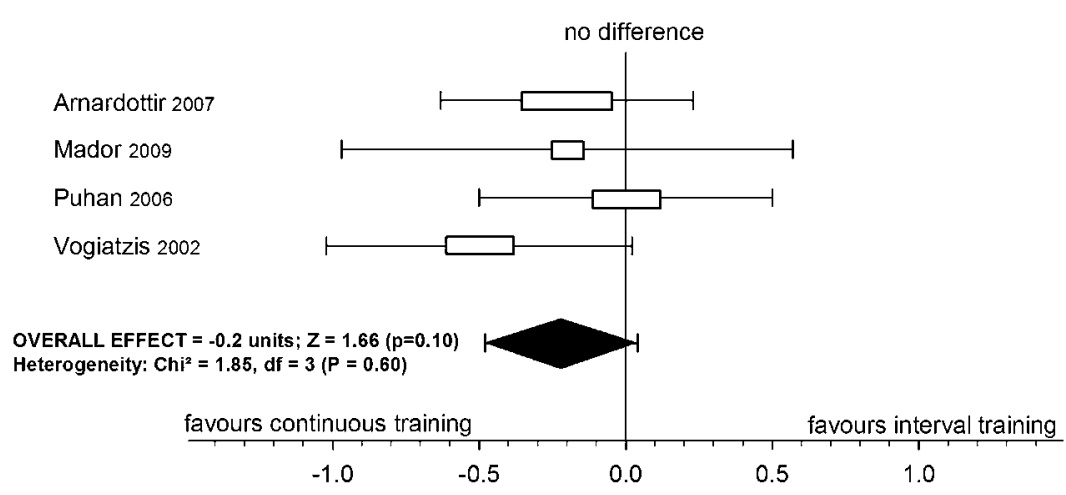

difference in change in chronic respiratory questionnaire: dyspnea 
Table 3 Results of meta-analyses for interval versus continuous exercise training

\begin{tabular}{|c|c|c|c|c|}
\hline Outcome & Interval (95\% Cl) & Continuous $(95 \% \mathrm{Cl})$ & WMD (95\% Cl) & $\begin{array}{l}\text { No. of participants in interval: } \\
\text { continuous (studies) }\end{array}$ \\
\hline IET: $\mathrm{VO}_{2 \text { peak }}(1 / \mathrm{min})$ & $0.80(-0.01$ to 0.16$)$ & $0.12(0.03$ to 0.20$)$ & $-0.04(-0.13$ to 0.05$)$ & $102: 112(6)$ \\
\hline IET: lactate threshold (I/min) & $0.10(0.03$ to 0.17$)$ & $0.09(0.04$ to 0.13$)$ & $0.01(-0.05$ to 0.07$)$ & $63: 63(4)$ \\
\hline IET isotime: $V_{E}(1 / m i n)$ & $-3(-7$ to 1$)$ & $-2(-7$ to 2$)$ & $-1(-5$ to 3$)$ & $65: 70(4)$ \\
\hline IET isotime: dyspnoea (Borg 1-10) & $-1.6(-2.5$ to -0.7$)$ & $-1.3(-2.5$ to 0$)$ & $-0.30(-1.02$ to 0.41$)$ & $73: 81(4)$ \\
\hline IET isotime: breathing frequency (breaths/min) & $-3(-5$ to -2$)$ & $-3(-5$ to -1$)$ & $0(-2$ to 2$)$ & $73: 81(4)$ \\
\hline 6MWT: distance (metres) & $48(29$ to 68$)$ & 42 (25 to 59$)$ & $4(-14$ to 23$)$ & $110: 118(4)$ \\
\hline CRO: dyspnoea & $1.0(0.6$ to 1.4$)$ & $1.2(0.7$ to 1.7$)$ & $-0.2(-0.5$ to 0.0$)$ & $106: 112(4)$ \\
\hline CRO: total & $0.7(0.5$ to 1.0$)$ & $0.8(0.6$ to 1.1$)$ & $-0.1(-0.3$ to 0.2$)$ & 79:83 (3) \\
\hline HAD: anxiety & $-2.0(-2.9$ to -1.1$)$ & $-2.2(-3.3$ to -1.2$)$ & $0.3(-0.8$ to 1.3$)$ & $68: 73(2)$ \\
\hline HAD: depression & $-1.9(-2.9$ to -0.9$)$ & $-2.2(-3.7$ to -0.8$)$ & $0.5(-0.5$ to 1.5$)$ & $72: 76(2)$ \\
\hline
\end{tabular}

CRQ, Chronic Respiratory Questionnaire; HAD, Hospital Anxiety and Depression scale; IET, incremental exercise test; $6 \mathrm{MWT}, 6$ minute walk test; $\mathrm{V}_{\mathrm{E}}$, minute ventilation; $\mathrm{VO}_{2 \text { peak, }}$ peak oxygen uptake; WMD, weighted mean difference.

interval durations (20/40 and $30 \mathrm{~s}$ intervals, respectively), it is possible that interval exercise of $<1 \mathrm{~min}$ at $\geq 100 \%$ of $\mathrm{P}_{\text {peak }}$ is better tolerated than high intensity continuous exercise.

It is well established that pulmonary rehabilitation, including endurance exercise training, results in significant and clinically meaningful improvements in health-related quality of life. ${ }^{3}$ While pooled results for the CRQ total score and HAD subscales were not different between continuous and interval training, the common effect for CRQ dyspnoea showed a trend in favour of continuous training. The minimally important difference of 0.5 for this measure ${ }^{24}$ also falls within the lower end of the confidence interval, suggesting a larger benefit from continuous training. Therefore, this review has not ruled out the possibility that continuous training is more effective in changing the CRQ dyspnoea score than interval exercise.

Limitations of the meta-analysis include the heterogeneity of the training protocols as well as small sample sizes in the primary trials. The duration of the intervals ranged from $30 \mathrm{~s}$ to 3 min of high intensity exercise. Rehabilitation programme lengths ranged from 3 to 16 weeks and training frequencies ranged from two to five times per week. Likewise, there was a wide range in the intensity and duration of continuous training protocols. However, despite the heterogeneous nature of the training parameters, the effects were similar, suggesting that differences in adjusting several training parameters are unlikely to have a major effect on exercise performance and quality of life. ${ }^{10}$ In addition, heterogeneity as measured by the $\mathrm{I}^{2}$ statistic suggested that a low percentage of the variability in effect estimates was due to heterogeneity rather than chance sampling error. Other study design limitations included the lack of blinding of outcome assessors, high drop-out rates and poor concealment of allocation.

In conclusion, evidence from the eight trials included in this review suggests that there are no differences between the effect of interval and continuous training on improving exercise capacity and health-related quality of life in individuals with COPD. Interval training may therefore be considered as a training option or complement to continuous exercise in patients unable to tolerate high intensity continuous exercise. Patient preference should also be considered in choice of protocol as it may impact adherence to treatment. Studies of high methodological quality with large numbers of participants are required to establish which types of interval training protocols are most efficacious and to evaluate the response to training in specific subgroups of patients, particularly in those with greater disease severity.
Acknowledgements We would like to thank the authors of the primary studies for their responses to our information requests.

Funding DB is supported by a Canada Research Chair, MB by the Ontario Respiratory Care Society and the Canadian Respiratory Health Professionals, and RG by the University of Toronto NSA Chair in Respiratory Rehabilitation Research. Other funders: CIHR, Ontario Respiratory Care Society, Canadian Respiratory Health Professionals and the University of Toronto.

Competing interests None.

Provenance and peer review Not commissioned; externally peer reviewed.

\section{REFERENCES}

1. Lopez AD, Shibuya K, Rao C, et al. Chronic obstructive pulmonary disease: current burden and future projections. Eur Respir J 2006;27:397-412.

2. Wouters EF, Creutzberg EC, Schols AM. Systemic effects in COPD. Chest 2002;121:127S-30S

3. Lacasse $\mathbf{Y}$, Wong $\mathrm{E}$, Guyatt $\mathrm{GH}$, et al. Meta-analysis of respiratory rehabilitation in chronic obstructive pulmonary disease. Lancet 1996;348:1115-9.

4. Ries AL, Bauldoff GS, Carlin BW, et al. Pulmonary rehabilitation: Joint ACCP/AACVPR evidence-based clinical practice guidelines. Chest 2007;131:4S-42S.

5. Casaburi R, Patessio A, loli F, et al. Reductions in exercise lactic acidosis and ventilation as a result of exercise training in patients with obstructive lung disease. Am Rev Respir Dis 1991;143:9-18.

6. Maltais F, LeBlanc $P$, Jobin J, et al. Intensity of training and physiologic adaptation in patients with chronic obstructive pulmonary disease. Am J Respir Crit Care Med 1997; 155:555-61.

7. Punzal PA, Ries AL, Kaplan RM, et al. Maximum intensity exercise training in patients with chronic obstructive pulmonary disease. Chest 1991;100:618-23.

8. Arnardottir RH, Boman G, Larsson K, et al. Interval training compared with continuous training in patients with COPD. Respir Med 2007:101:1196-204.

9. Coppoolse R, Schols AM, Baarends EM, et al. Interval versus continuous training in patients with severe COPD: a randomized clinical trial. Eur Respir J 1999;14: 258-63.

10. Mador MJ, Krawza M, Alhajhusain A, et al. Interval training versus continuous training in patients with chronic obstructive pulmonary disease. J Cardiopulm Rehabil Prev 2009;29:126-32.

11. Puhan MA, Busching G, Schunemann HJ, et al. Interval versus continuous highintensity exercise in chronic obstructive pulmonary disease: a randomized trial. Ann Intern Med 2006;145:816-25.

12. Sabapathy S, Kingsley RA, Schneider DA, et al. Continuous and intermittent exercise responses in individuals with chronic obstructive pulmonary disease. Thorax 2004;59:1026-31.

13. Varga J, Porszasz J, Boda K, et al. Supervised high intensity continuous and interval training vs. self-paced training in COPD. Respir Med 2007;101:2297-304.

14. Vogiatzis I, Nanas S, Kastanakis E, et al. Dynamic hyperinflation and tolerance to interval exercise in patients with advanced COPD. Eur Respir J 2004;24 385-90.

15. Vogiatzis I, Nanas $\mathbf{S}$, Roussos $\mathrm{C}$. Interval training as an alternative modality to continuous exercise in patients with COPD. Eur Respir J 2002;20:12-9.

16. Vogiatzis I, Terzis G, Nanas S, et al. Skeletal muscle adaptations to interval training in patients with advanced COPD. Chest 2005;128:3838-45.

17. Poole DC, Gaesser GA. Response of ventilatory and lactate thresholds to continuous and interval training. J Appl Physiol 1985;58:1115-21.

18. Laursen PB, Jenkins DG. The scientific basis for high-intensity interval training optimising training programmes and maximising performance in highly trained endurance athletes. Sports Med 2002;32:53-73. 
19. Wisloff U, Stoylen A, Loennechen JP, et al. Superior cardiovascular effect of aerobic interval training versus moderate continuous training in heart failure patients: a randomized study. Circulation 2007:115:3086-94.

20. Nasis IG, Vogiatzis I, Stratakos G, et al. Effects of interval-load versus constantload training on the BODE index in COPD patients. Respir Med 2009;103:1392-98.

21. Celli BR, MacNee W. Standards for the diagnosis and treatment of patients with COPD: a summary of the ATS/ERS position paper. Eur Respir J 2004;23:932-46.

22. Jadad AR, Moore RA, Carroll D, et al. Assessing the quality of reports of randomized clinical trials: is blinding necessary? Control Clin Trials 1996:17:1-12.

23. Maher CG, Sherrington C, Herbert RD, et al. Reliability of the PEDro scale for rating quality of randomized controlled trials. Phys Ther 2003;83:713-21.

24. Schunemann HJ, Puhan M, Goldstein R, et al. Measurement properties and interpretability of the Chronic respiratory disease questionnaire (CRO). Copd 2005;2:81-9.

25. Redelmeier DA, Bayoumi AM, Goldstein RS, et al. Interpreting small differences in functional status: the Six Minute Walk test in chronic lung disease patients. Am J Respir Crit Care Med 1997;155:1278-82.
26. Krauza M, Alhajhusain A, Khan A, et al. Interval compared with continuous exercise training in patients with COPD. Am J Crit Care Med 2006;173:220.

27. Meyer K, Samek L, Schwaibold M, et al. Interval training in patients with severe chronic heart failure: analysis and recommendations for exercise procedures. Med Sci Sports Exerc 1997;29:306-12.

28. Ambrosino N, Strambi S. New strategies to improve exercise tolerance in chronic obstructive pulmonary disease. Eur Respir J 2004;24:313-22.

29. Swain DP, Franklin BA. VO(2) reserve and the minimal intensity for improving cardiorespiratory fitness. Med Sci Sports Exerc 2002;34:152-7.

30. Palange $\mathbf{P}$, Ward SA, Carlsen $\mathrm{KH}$, et al. Recommendations on the use of exercise testing in clinical practice. Eur Respir J 2007;29:185-209.

31. Casaburi R. Factors determining constant work rate exercise tolerance in COPD and their role in dictating the minimal clinically important difference in response to interventions. COPD 2005;2:131-6.

32. Laviolette L, Bourbeau J, Bernard S, et al. Assessing the impact of pulmonary rehabilitation on functional status in COPD. Thorax 2008;63:115-21. 